\title{
微量元素与朝鲜淫羊蕉中黄酮对原代成骨细胞增殖和 分化的拮抗与协同效应
}

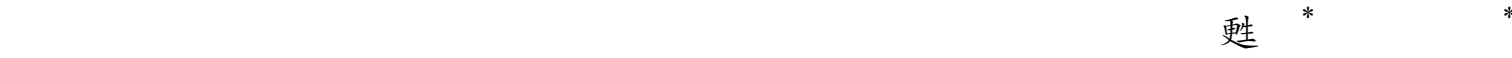 \\ (1) 沈阳药科大学天然药物化学教研室, 沈阳 110016; (2) 香港城市大学深圳研究院药用生物芯片重点实验室, 深圳 518057; \\ (3) 香港城市大学生物及化学系, 香港; (4) 河北大学化学与环境科学学院, 保定 071002. \\ * 联系人, E-mail: bhmyang@(cityu.edu.hk; yaoxinsheng@vip.tom.com)
}

\begin{abstract}
摘要＼cjkstart研究了淫羊蕉总黄酮、淫羊蕉苷与淫羊㺃中含量较高的微量元素合用以及单独应用对原代成骨 细胞增殖以及分化的影响, 并通过统计学分析以期发现是否存在潜在的协同、拮抗与加和效应. 结果表

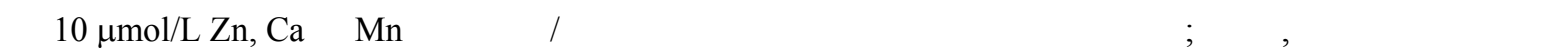
胞的分化. 此外, 0.1 和 $1 \mu \mathrm{mol} / \mathrm{L} \mathrm{Zn}$ 与 $10 \mu \mathrm{mol} / \mathrm{L}$ 淫羊藿苷, $10 \mu \mathrm{mol} / \mathrm{L} \mathrm{Mn}$ 与 $0.06 \mu \mathrm{g} / \mathrm{mL}$ 总黄酮合用对 成骨细胞的增殖显示出较强的抑制效应. 同时, 在某些淫羊雚苷 $-\mathrm{Zn} / \mathrm{Mn}$, 总黄酮 $-\mathrm{Zn} / \mathrm{Ca} / \mathrm{Mn}$ 合用时, 对 成骨细胞的分化也显示出一定的抑制效应. 结果显示, 3 种矿物元素与淫羊蕉苷、总黄酮的某些组合可 以有效地提高淫羊蕉苷、总黄酮对原代成骨细胞增殖和分化的作用.
\end{abstract}

\section{关键词 淫羊雚 淫羊嚾苷 总黄酮 钙 锌 锰 成骨细胞 增殖 分化 协同作用}

骨质疏松是一种以骨密度降低、骨组织微结构破 坏为特征, 从而导致骨骼脆性增加和易发生骨折的全 身性骨骼病患. 随着人口老龄化社会的到来, 骨质疏松 及其引起的骨折构成了对公共健康的巨大威胁. 动物 实验表明, 淫羊嚄等中药对骨质疏松和骨折具有一定 的预防和治疗作用 $[1 \sim 3]$. 中药淫羊蒦含有黄酮、植物甾 醇等多种药用活性成分，在心血管疾病、关节炎、不孕 不育、腰滕酸软、肢体麻木无力等方面已经有近千年的 应用 ${ }^{[4]}$. 近年来许多研究表明淫羊雚在骨质疏松的治 疗方面显示出一定的潜力, 因此, 淫羊蕉在治疗骨质疏 松的复方中得到广泛应用 $[-7]$. 但是, 绝大多数研究都 是集中于淫羊嚾中的黄酮成分. 实验表明, 淫羊蕉粗提 物、总黄酮、以及主要黄酮成分(Epimedium brevicornum Maxim, E. koreanum Nakai)显示一定的促进成骨细胞 [8 10]以及成骨细胞样细胞UMR-106 的增殖活性 ${ }^{[11,12]}$.

近年来, 营养素对骨生理的影响引起人们的广 泛兴趣. 这些营养素包括: 矿物质, 如钙、镁、锌、 铜、镇和硅; 维生素, 如维生素 $\mathrm{C} 、$ 维生素K和维生素 $\mathrm{A}$; 以及常量营养物, 如蛋白质、脂肪酸和糖等 ${ }^{[13]}$. 矿物元素在一些基本的生化代谢过程中发挥一定的 作用, 而且矿物元素生化重要性的研究也随之增加. 如钙在各种细胞信号传递过程中发挥信使的作用.
在骨骼与外周血之间存在着钻离子的稳态平衡, 且 血清中钙离子浓度保持在 $1 \mathrm{mg} / \mathrm{L}$ 水平 ${ }^{[14]}$. 钻的摄入 与上述钻平衡存在密切关系, 而钻补充制剂可以有 效减缓绝经期后期妇女脊椎以及四肢骨的骨丢失 ${ }^{[15]}$. 锌和铜作为酶辅助因子促进骨矿化以及骨胶原结构 的形成, 从而在结缔组织代谢中发挥重要作用 ${ }^{[16]}$. 很多研究显示, 锌补充疗法可以增强人体免疫功能, 促进性成熟, 缓解氧化损伤 117$]$. 但是在体外锌抑制 成骨细胞的分化从而影响矿化组织的形成 ${ }^{[18]}$. 近来 基于老年健康受试者的一项临床研究显示出骨质疏 松与锌缺乏之间的临床关系 ${ }^{[19]}$. 锰作为酶的组分或 激活物在一系列生理过程中发挥重要作用, 而且锰 缺乏可以引起许多物种动物的骨发育异常. 如锰是 糖基转移酶的首选辅助因子, 而糖基转移酶在合成 正常软骨与骨骼形成中发挥重要的作用 ${ }^{[20]}$.

此外, 中药中含有多种微量元素, 而且其含量具 有一定的潜在生物效应. 然而中药中的微量元素并 没有引起足够的重视, 目前的研究多是分别研究化 学成分与微量元素的有效性. 本文中, 我们选择 3 种 淫羊嚄中含量较高的矿物元素: $\mathrm{Ca}, \mathrm{Zn}, \mathrm{Mn}$, 来研究 微量元素是否以及如何影响总黄酮、淫羊蒦苷对成骨 细胞增殖和分化的效应. 


\section{1 实验}

(i) 材料. 朝鲜淫羊嚄 (E. koreanum Nakai) 药 材于 2003 年 6 7 月份采集于辽宁新宾, 并经过沈阳 药科大学生药学专业孙启时教授鉴定. 样本 (No. 19980816-1)保存在深圳中药及天然药物研究中心标 本室.

新生 $\mathrm{NIH}$ 小鼠购自广州中医药大学实验动物中 心, SPF 级, 合格证号: SCXK 2003-0001. 胰酶、胎牛 血清购自 Gibco. MTT(噻唑蓝)、17及-雌二醇、青霉素、 链霉素、I型胶原酶和 DMEM 培养基(无酚红)购自 Sigma. 碱性磷酸酶活力试剂盒购自南京建成生物工 程研究所, 蛋白含量试剂盒购自碧云天生物技术研 究所. 氯化锌、氯化钙、氯化锰以及其他试剂均为分 析纯.

(ii ) 测试样品的配制. 淫羊藿苷和总黄酮按照 文献方法制备 [21]. 淫羊蕉苷和总黄酮以二甲基亚砜 (DMSO)分别配成 $10 \mathrm{mmol} / \mathrm{L}, 6 \mathrm{mg} / \mathrm{mL}$ 咜备液, 临用 前以培养基稀释到工作液浓度. $\mathrm{NaF}$ 和 $17 \beta$-雌二醇分 别以PBS和无水乙醇配成 $10 \mathrm{mmol} / \mathrm{L}$ 䛎备液, 临用前 以培养基稀释作为阳性对照. $\mathrm{ZnCl}_{2}, \mathrm{CaCl}_{2}$ 以及 $\mathrm{MnCl}_{2}$ 溶解方式同 $\mathrm{NaF}$. 为了避免 $\mathrm{DMSO}$ 和乙醇对实验结果 的影响，严格控制其含量在 $0.1 \%$ (体积比)以下，同时在 对照组添加同样浓度的DMSO和无水乙醇.

(iii) 原代成骨细胞的分离. 原代成骨细胞按照 文献 [22]方法, 采用酶系列消化方法从新生 $24 \mathrm{~h} \mathrm{NIH}$ 乳鼠领盖骨制备. 即无菌条件下分离出䏠盖骨, 以手 术刀刮去骨内外膜. 将骨片切成 $1 \sim 2 \mathrm{~mm}^{2}$ 大小碎片,

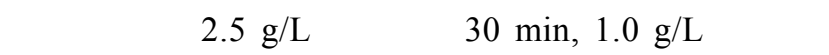
原酶消化 2 次各 $1 \mathrm{~h}$. 收集胶原酶消化下来的细胞于 含 $10 \%$ 血清, $100 \mathrm{U} / \mathrm{mL}$ 青霉素, $100 \mu \mathrm{g} / \mathrm{mL}$ 链霉素的 DMEM培养基中, 在 $37^{\circ} \mathrm{C}, 5 \% \mathrm{CO}_{2}$ 条件下培养 $24 \mathrm{~h}$, 换液. 取第 2 4 代细胞, 待细胞生长汇合 $70 \%$, 以 $0.25 \%$ 胰酶消化后, 调整细胞密度 $2.5 \times 10^{4}$ 个 $/ \mathrm{cm}^{2}$ 接 种于 96 孔及 48 孔培养板中 $\left(0.28 \mathrm{~cm}^{2} /\right.$ 孔, $0.95 \mathrm{~cm}^{2} /$ 孔) 在 $10 \%$ 血清, $100 \mathrm{U} / \mathrm{mL}$ 青霉素, $100 \mu \mathrm{g} / \mathrm{mL}$ 链霉素, $37^{\circ} \mathrm{C}, 5 \% \mathrm{CO}_{2}$ 条件下继续培养. 黄酮组分(淫羊嚄苷 $0.1 \sim 10 \mu \mathrm{mol} / \mathrm{L}$, 总黄酮 $0.06 \sim 6 \mu \mathrm{g} / \mathrm{mL}$ )与 3 种元素 $\left(\mathrm{ZnCl}_{2} / \mathrm{CaCl}_{2} / \mathrm{MnCl}_{2} \quad 0.1 \sim 10 \mu \mathrm{mol} / \mathrm{L}\right)$ 为临用前配制, 合用时同时加入. 为了排除类固醇成分对增殖的影 响, 选用不含有酚红的培养基添加医用活性碳处理 过的血清.

（iv）测定样品对成骨细胞增殖的影响. 采用噻
唑蓝(MTT)方法研究药物对成骨细胞增殖的影响 ${ }^{[23]}$. 具体如下: 将成骨细胞以 $2.5 \times 10^{4}$ 个 $/ \mathrm{cm}^{2}$ 接种于 96 孔培养板中, 静置 $24 \mathrm{~h}$ 后加药, 随后继续培养 $48 \mathrm{~h}$. 实验结束前 $4 \mathrm{~h}$ 向培养板中加入MTT $(20 \mu \mathrm{L}, 5 \mathrm{mg} / \mathrm{mL})$, 培养 $4 \mathrm{~h}$. 实验结束去掉培养基, 每孔加入 $100 \mu \mathrm{L}$ DMSO溶解甲肤，随后在酶标仪上测定 $570 \mathrm{~nm}$ 吸光度， 参考波长 $655 \mathrm{~nm}$ (Bio-rad Model 680, USA).

( v ) 测定药物成骨细胞碱性磷酸酶(ALP)活性 的影响. 在测定碱性磷酸酶活力以及总蛋白含量实 验中, 成骨细胞以 $2.5 \times 10^{4}$ 个 $/ \mathrm{cm}^{2}$ 接种于 48 孔培养 板中, 加药后作用 $3 \mathrm{~d}$. 随后经 $-80^{\circ} \mathrm{C}$ 反复冻融同时超 声裂解细胞. 每孔裂解液分别按照碱性磷酸酶活力 试剂盒以及蛋白含量试剂盒方法测定碱性磷酸酶活 力以及总蛋白含量. 实验结果以蛋白含量归一化. 酶 活力单位定义: $1 \mathrm{~g}$ 组织蛋白在 $37^{\circ} \mathrm{C}$ 与底物作用 15 $\min$ 产生 $1 \mu \mathrm{g}$ 对硝基苯酚定义为一个酶活力单位.

(vi) 统计分析. 所有数据来自至少 3 次独立实 验, 采用平均值 \pm 标准偏差表示. 数据的组内比较采 用方差分析(ANOVA). 组间比较采用双因素方差分 析方法. $P<0.05$ 认为具有显著性差异.

\section{2 结果}

\section{1 淫羊蕉苷及总黄酮对原代成骨细胞增殖的影响}

如图 1 所示, 阳性对照 $1.0 \times 10^{-6} \mathrm{~mol} / \mathrm{L} 17 \beta$-雌二 醇对原代成骨细胞的增殖促进率为 $13 \%$, 而淫羊蕉 甘(Icariin) 与总黄酮 (TF) 在实验浓度范围内 $(1.0 \times$ $10^{-9} \sim 2.0 \times 10^{-5} \mathrm{~mol} / \mathrm{L}$ 和 $6 \times 10^{-7} \sim 1.2 \times 10^{-2} \mathrm{mg} / \mathrm{mL}$ ) 对 原代成骨细胞的增殖则显示出一定的抑制作用. 淫

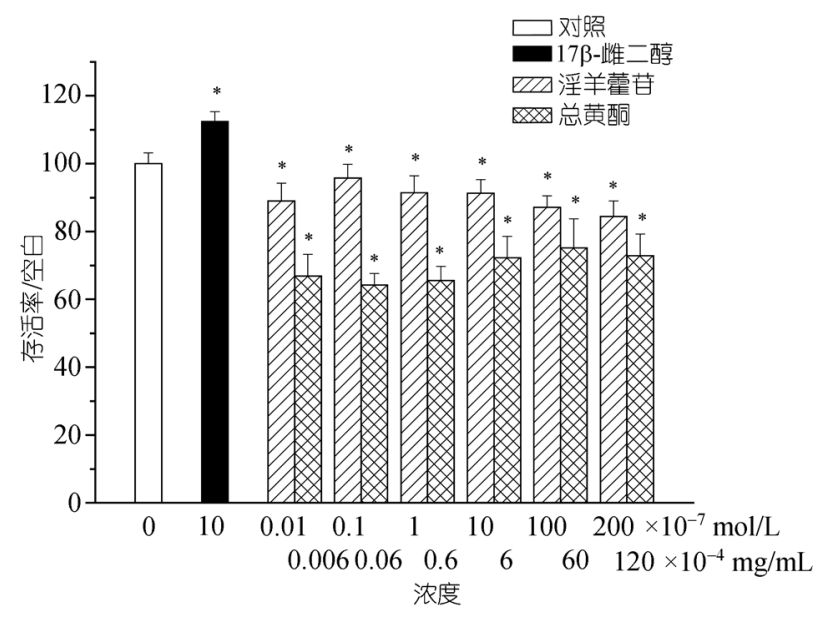

图 1 黄酮成分抑制原代成骨细胞的增殖 $n=5$, * 表示与空白对照组比, $P<0.05$ 
羊蕉苷与总黄酮的平均抑制率分别在 $15 \%$ 和 $30 \%$ 左 右. 假定黄酮苷的平均分子量为 600 , 那么 $1.2 \times 10^{-2}$ $\mathrm{mg} / \mathrm{mL}$ 和 $6.0 \times 10^{-3} \mathrm{mg} / \mathrm{mL}$ 总黄酮相当于 $2.0 \times 10^{-5}$ 和 $1.0 \times 10^{-5} \mathrm{~mol} / \mathrm{L}$ 淫羊嚾苷. 据此分析, 在同样的浓度 下, 总黄酮比淫羊蕉苷显示出更强的抑制作用.

\section{2 微量元素对成骨细胞增殖的影响}

$1.0 \times 10^{-6} \mathrm{~mol} / \mathrm{L}$ 氟化钠对原代成骨细胞的增殖 显示出 $18 \%$ 的促进率(图 2). 3 种淫羊雚中含量最高的 矿物元素 $(\mathrm{Ca}, \mathrm{Zn}, \mathrm{Mn})$ 与黄酮成分一样对成骨细胞的 增殖显示出一定的抑制作用. $\mathrm{Zn}$ 与 $\mathrm{Ca}$ 的抑制效应很 相似, 其平均抑制率约 $15 \%$; 但是, 在 $2.0 \times 10^{-5} \mathrm{~mol} / \mathrm{L}$ 与对照组比较没有统计学差异. $\mathrm{Zn}$ 和 $\mathrm{Mn}$ 相比, 相同浓 度下 $\mathrm{Mn}$ 显示出更强的抑制效应. 此外, 在 $1.0 \times 10^{-9} \sim$ $1.0 \times 10^{-5} \mathrm{~mol} / \mathrm{L}$ 浓度范围, 钙、锌和锰显示出持续的 抑制效应但没有剂量依赖性.

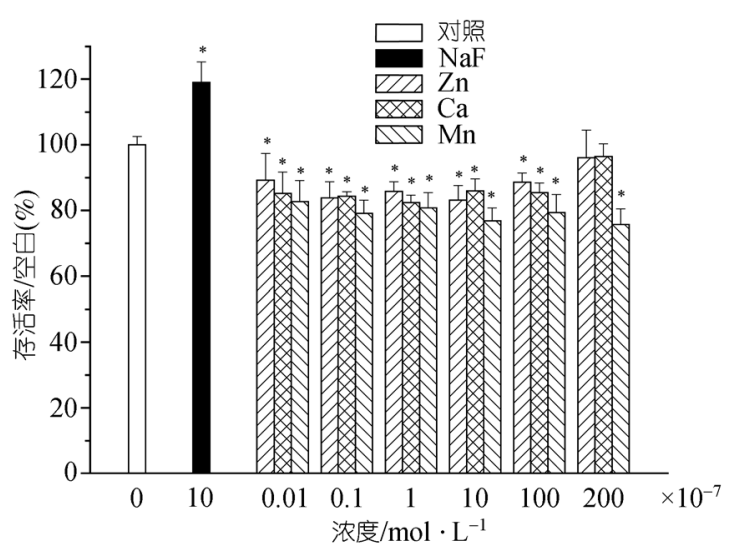

图 2 微量元素抑制原代成骨细胞的增殖 $n=5, *$ 表示与空白对照组比, $P<0.05$

2.3 淫羊蕉苷+微量元素及总黄酮+微量元素对原代 成骨细胞增殖的影响

为了研究微量元素与黄酮合用是否可以提高黄 酮的有效性, 我们研究了微量元素与黄酮合用对原 代成骨细胞增殖的影响, 旨在分析其是否存在潜在 的协同、加和或拮抗作用. 如图 3 中数据所示, 10 $\mu \mathrm{mol} / \mathrm{L} \mathrm{Zn,} \mathrm{Ca}$ 和 $\mathrm{Mn}$ 与黄酮合用与分别单独使用比 较对成骨细胞的增殖显示出较强的拮抗作用. 例如, $0.1 \mu \mathrm{mol} / \mathrm{L}, 1 \mu \mathrm{mol} / \mathrm{L}$ 淫羊雚苷, $10 \mu \mathrm{mol} / \mathrm{L} \mathrm{Zn} / \mathrm{Ca} / \mathrm{Mn}$ 单独作用时成骨细胞存活率为 $89.9 \%$ (淫羊藿苷 0.1 $\mu \mathrm{mol} / \mathrm{L}$ )， $88.7 \%$ (淫羊藿苷 $1 \mu \mathrm{mol} / \mathrm{L}$ )， $88.6 \%(\mathrm{Zn})$, $85.5 \%(\mathrm{Ca})$ 和 $79.3 \%(\mathrm{Mn})$, 而其合用 $48 \mathrm{~h}$ 后成骨细胞 的存活率提高到 $96.7 \%, 93.0 \%, 104.0 \%, 105.6 \%$,
105.3\%, 101.7\% (图 3(a), (c) (e)). 同样, $10 \mu \mathrm{mol} / \mathrm{L} \mathrm{Zn}$ 和 $\mathrm{Ca}$ 与 $0.06,0.6$ 和 $6 \mu \mathrm{g} / \mathrm{mL}$ 总黄酮共同作用 $48 \mathrm{~h}$, 成骨细胞的存活率为 $97.1 \%, 102.7 \%, 106.8 \%, 100.3 \%$, $98.2 \%$ 和 $98.4 \%$; 而其单独应用时的存活率分别为 88.6\%(Zn), 85.5\%(Ca), 66.1\%(总黄酮 $0.06 \mu \mathrm{g} / \mathrm{mL}$ ), $72.2 \%$ (总黄酮 $0.6 \mu \mathrm{g} / \mathrm{mL}$ ) 和 $73.9 \%$ (总黄酮 $6 \mu \mathrm{g} / \mathrm{mL}$ ) (图 3(b)和(d)). 此外, 拮抗作用也出现在其他的一些 淫羊蒦苷 $-\mathrm{Zn} / \mathrm{Ca} / \mathrm{Mn}$ 和总黄酮-Ca 的合用中. 与此相 反, $0.1 \mu \mathrm{mol} / \mathrm{L}, 1 \mu \mathrm{mol} / \mathrm{L} \mathrm{Zn}$ 与 $10 \mu \mathrm{mol} / \mathrm{L}$ 淫羊藿苷合 用, $10 \mu \mathrm{mol} / \mathrm{L} \mathrm{Mn}$ 与 $0.06 \mu \mathrm{g} / \mathrm{mL}$ 总黄酮合用, 显示出 更强的抑制效应: $71.5 \%, 67.5 \%, 58.1 \%(85.8 \% \mathrm{Zn}$ $(0.1 \mu \mathrm{mol} / \mathrm{L}), 83.2 \% \mathrm{Zn}(1 \mu \mathrm{mol} / \mathrm{L})$; 图 3(a)和(f)).

2.4 淫羊蕉苷及总黄酮对原代成骨细胞分化过程中 碱性磷酸酶活性的影响

如图 4 所示, $1.0 \times 10^{-6} \mathrm{~mol} / \mathrm{L} \quad 17 \beta$-雌二醇对原代 成骨细胞分化过程中碱性磷酸酶活力的促进率达 $56.2 \%$. 同样, 在不同浓度的淫羊蕉苷以及总黄酮对原 代成骨细胞的分化过程中碱性磷酸酶活力均显示出不 同程度的促进效应. 在 $1.0 \times 10^{-7} \sim 1.0 \times 10^{-5} \mathrm{~mol} / \mathrm{L}$ 浓 度下, 淫羊嚄苷比总黄酮对原代成骨细胞的分化显示 出更强的促进作用. 值得注意的是, 淫羊嚄苷在 $1.0 \times$ $10^{-9}, 1.0 \times 10^{-7}, 1.0 \times 10^{-6} \mathrm{~mol} / \mathrm{L}$ 浓度下对成骨细胞碱 性磷酸酶活力的促进率高达 2.1, 3.8 和 4.8 倍.

2.5 微量元素对原代成骨细胞分化过程中碱性磷酸 酶活力的影响

微量元素对成骨细胞分化中碱性磷酸酶活力的 影响见图 5. $1.0 \times 10^{-9} \sim 1.0 \times 10^{-5} \mathrm{~mol} / \mathrm{L} 3$ 种矿物元素 显著促进碱性磷酸酶活力, 但是 $1.0 \times 10^{-6} \mathrm{~mol} / \mathrm{L} \mathrm{Mn}$ 例外. $\mathrm{Zn}, \mathrm{Ca}$ 和 $\mathrm{Mn}$ 的最大促进率分别出现在 $1.0 \times$ $10^{-8}, 1.0 \times 10^{-7}$ 和 $1.0 \times 10^{-5} \mathrm{~mol} / \mathrm{L}$, 达到 $2.3,3.4$ 和 2.1 倍(图 5).

2.6 淫羊嚄苷+微量元素以及总黄酮+微量元素对成 骨细胞分化过程中碱性磷酸酶活力的影响

黄酮与 3 种矿物元素分别作用 3 天引起成骨细胞 分化过程中碱性磷酸酶活力的显著增强(图 4 和 5). 而 与单独应用比较, 微量元素的存在非常显著地提高了 淫羊蕉苷 $\left(1.0 \times 10^{-7} \sim 1.0 \times 10^{-5} \mathrm{~mol} / \mathrm{L}\right)$ 和总黄酮 $(0.06 \sim 6$ $\mu \mathrm{g} / \mathrm{mL})$ 对成骨细胞分化的作用. 如图 6 所示, 10 $\mu \mathrm{mol} / \mathrm{L} \mathrm{Zn,} \mathrm{Ca}$ 和 Mn 与淫羊藿苷/总黄酮合用对成骨 细胞的分化显示较强的协同作用, 而且这一结果与 前面对成骨细胞增殖的结果具有相关性(图 3). Zn 显 
示出最明显的影响, 在多数 $\mathrm{Zn}$ 与淫羊雚苷/总黄酮的合 用中显示出不同程度的促进或抑制效应. 如 $10 \mu \mathrm{mol} / \mathrm{L}$

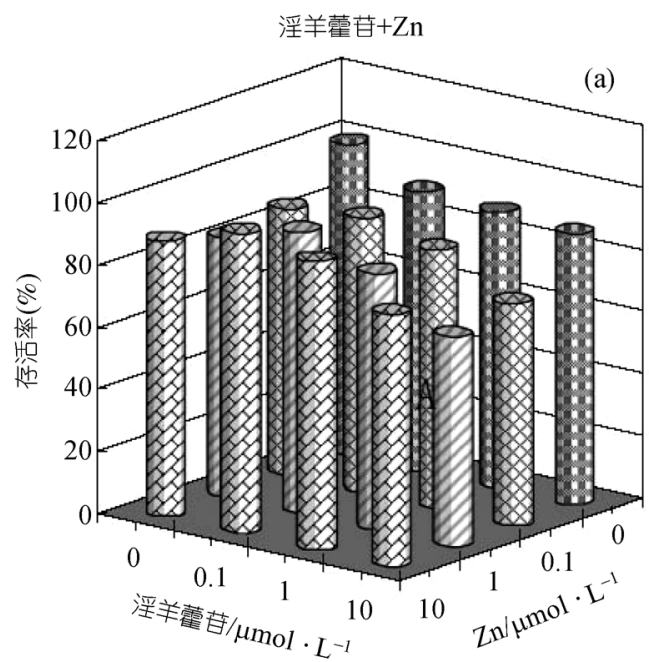

\begin{tabular}{|c|c|c|c|c|}
\hline \multicolumn{4}{|c|}{ 存活率(\%) } & $\mathrm{Zn}$ \\
\hline $100.0 \pm 3.2$ & $89.9 \pm 5.2^{*}$ & $88.7 \pm 4.1^{*}$ & $87.2 \pm 5.0^{*}$ & 0 \\
\hline $85.8 \pm 3.0^{*}$ & $88.3 \pm 7.6^{*}$ & $83.2 \pm 6.9^{*}$ & $71.5 \pm 5.1^{* \#}$ & 0.1 \\
\hline $83.2 \pm 4.4^{*}$ & $90.3 \pm 7.4^{*}$ & $82.5 \pm 3.8^{*}$ & $67.5 \pm 6.4^{* \#}$ & 1 \\
\hline $88.6 \pm 2.8^{*}$ & $96.7 \pm 8.1^{\#}$ & $93.0 \pm 8.6^{* \#}$ & $81.4 \pm 8.7^{* \#}$ & 10 \\
\hline 0 & 0.1 & 1 & 10 & $\begin{array}{l}\text { 淫羊藿苷 } \\
/ \mu \mathrm{mol} \cdot \mathrm{L}^{-1}\end{array}$ \\
\hline
\end{tabular}

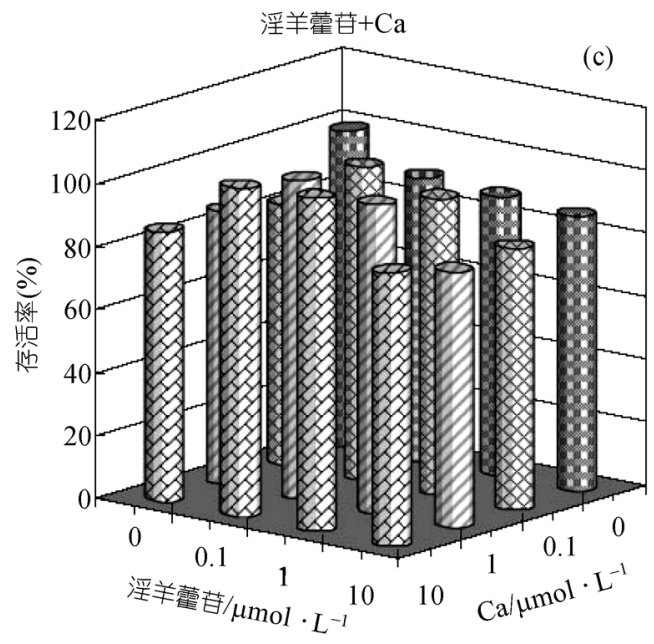

\begin{tabular}{|c|c|c|c|c|}
\hline \multicolumn{4}{|c|}{ 存活率(\%) } & $\begin{array}{c}\mathrm{Ca} \\
/ \mu \mathrm{mol} \cdot \mathrm{L}^{-1}\end{array}$ \\
\hline $100.0 \pm 1.2$ & $89.9 \pm 5.2^{*}$ & $88.7 \pm 4.1^{*}$ & $87.2 \pm 5.0^{*}$ & 0 \\
\hline $82.4 \pm 2.3^{*}$ & $99.2 \pm 2.9^{\#}$ & $93.9 \pm 7.2^{\#}$ & $82.8 \pm 6.6^{*}$ & 0.1 \\
\hline $86.0 \pm 3.6^{*}$ & $100.7 \pm 5.4^{\#}$ & $97.8 \pm 4.9^{\#}$ & $81.2 \pm 8.7^{*}$ & 1 \\
\hline $85.5 \pm 2.9^{*}$ & $104.0 \pm 8.0^{\#}$ & $105.6 \pm 8.0^{\#}$ & $86.7 \pm 10.3^{*}$ & 10 \\
\hline 0 & 0.1 & 1 & 10 & $\begin{array}{l}\text { 淫羊藿甘 } \\
/ \mu \mathrm{mol} \cdot \mathrm{L}^{-1}\end{array}$ \\
\hline
\end{tabular}

$\mathrm{Zn}$ 与淫羊蕉苷 $\left(1.0 \times 10^{-7} \sim 1.0 \times 10^{-5} \mathrm{~mol} / \mathrm{L}\right)$ 或总黄酮 (0.06 6 $\mu \mathrm{g} / \mathrm{mL})$ 合用提高碱性磷酸酶活力达 3.9, 5.8,

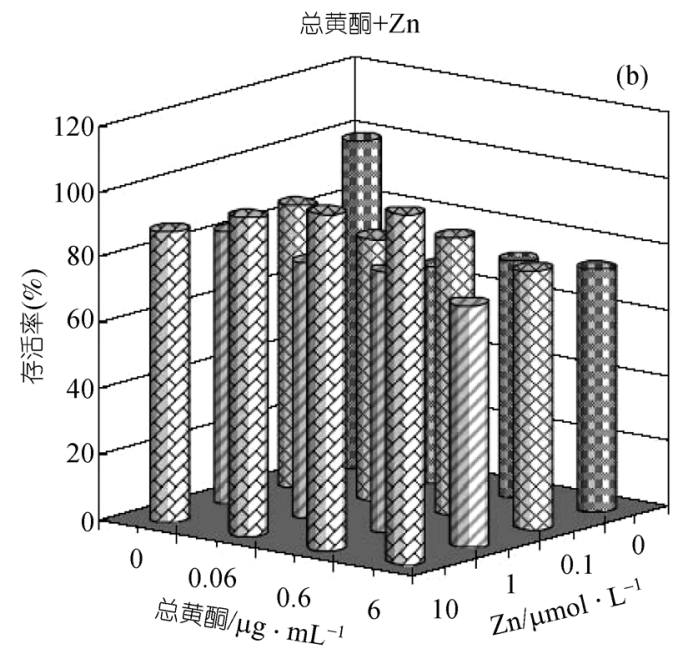

\begin{tabular}{|c|c|c|c|c|}
\hline \multicolumn{4}{|c|}{ 存活率(\%) } & $\mathrm{Zn}$ \\
\hline $100.0 \pm 5.8$ & $66.1 \pm 4.2^{*}$ & $72.2 \pm 3.4^{*}$ & $73.9 \pm 6.4^{*}$ & 0 \\
\hline $85.8 \pm 3.0^{*}$ & $80.0 \pm 6.0^{*}$ & $84.7 \pm 2.8^{*}$ & $79.0 \pm 6.3^{*}$ & 0.1 \\
\hline $83.2 \pm 4.4^{*}$ & $78.1 \pm 6.5^{*}$ & $79.5 \pm 2.6^{*}$ & $73.8 \pm 5.3^{*}$ & 1 \\
\hline $88.6 \pm 2.8^{*}$ & $97.1 \pm 4.3^{\#}$ & $102.7 \pm 6.5^{\#}$ & $106.8 \pm 4.7^{* \#}$ & 10 \\
\hline 0 & 0.06 & 0.6 & 6 & $\begin{array}{c}\text { 总黄酮 } \\
/ \mu \mathrm{g} \cdot \mathrm{mL}^{-1}\end{array}$ \\
\hline
\end{tabular}

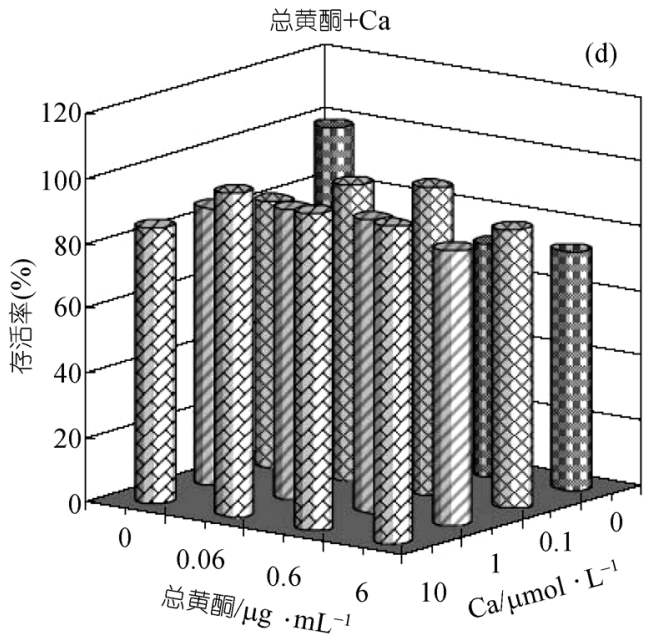

\begin{tabular}{|c|c|c|c|c|}
\hline \multicolumn{4}{|c|}{ 存活率(\%) } & $\begin{array}{c}\mathrm{Ca} \\
/ \mu \mathrm{mol} \cdot \mathrm{L}^{-1}\end{array}$ \\
\hline $100.0 \pm 4.5$ & $66.1 \pm 4.2^{*}$ & $72.2 \pm 3.4^{*}$ & $73.9 \pm 6.4^{*}$ & 0 \\
\hline $82.4 \pm 2.3^{*}$ & $91.7 \pm 2.3^{* \#}$ & $95.2 \pm 2.6^{* \#}$ & $86.2 \pm 5.4^{*}$ & 0.1 \\
\hline $86.0 \pm 3.6^{*}$ & $89.6 \pm 5.3^{* \#}$ & $90.6 \pm 2.2^{* \#}$ & $85.3 \pm 7.2^{*}$ & 1 \\
\hline $85.5 \pm 2.9^{*}$ & $100.3 \pm 3.2^{\#}$ & $98.2 \pm 6.9^{\#}$ & $98.4 \pm 5.0^{\#}$ & 10 \\
\hline 0 & 0.06 & 0.6 & 6 & $\begin{array}{c}\text { 总黄酮 } \\
/ \mu \mathrm{g} \cdot \mathrm{mL}^{-1}\end{array}$ \\
\hline
\end{tabular}



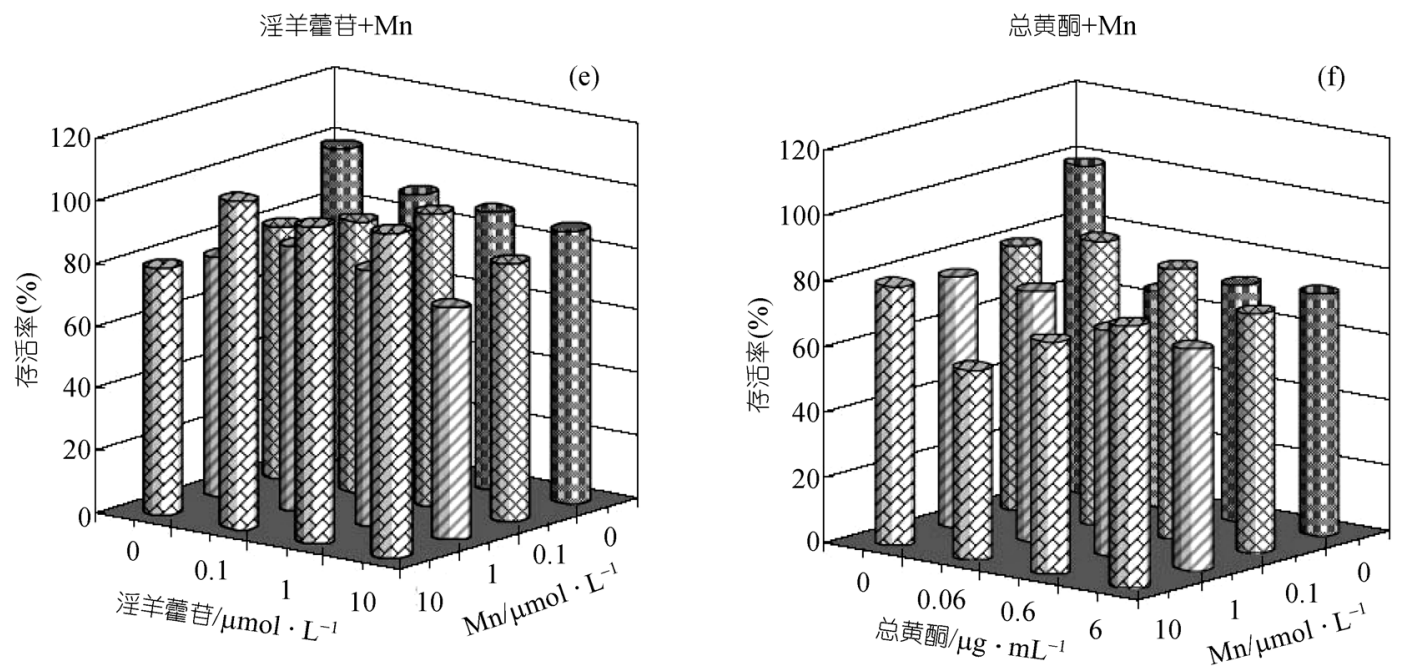

\begin{tabular}{|c|c|c|c|c|}
\hline \multicolumn{4}{|c|}{ 存活率(\%) } & $\begin{array}{c}\mathrm{Mn} \\
/ \mathrm{umol} \cdot \mathrm{L}^{-1}\end{array}$ \\
\hline $100.0 \pm 3.4$ & $89.9 \pm 5.2^{*}$ & $88.7 \pm 4.1^{*}$ & $87.2 \pm 5.0^{*}$ & 0 \\
\hline $80.8 \pm 4.6^{*}$ & $87.1 \pm 2.6^{*}$ & $94.0 \pm 8.9^{* \#}$ & $82.7 \pm 6.5^{*}$ & 0.1 \\
\hline $76.9 \pm 3.9^{*}$ & $84.8 \pm 4.6^{*}$ & $82.0 \pm 7.7^{*}$ & $74.5 \pm 6.2^{*}$ & 1 \\
\hline $79.3 \pm 5.6^{*}$ & $105.3 \pm 4.0^{\#}$ & $101.7 \pm 6.6^{\#}$ & $104.2 \pm 3.7^{* \#}$ & 10 \\
\hline 0 & 0.1 & 1 & 10 & $\begin{array}{l}\text { 淫羊雚苷 } \\
/ \mu \mathrm{mol} \cdot \mathrm{L}^{-1}\end{array}$ \\
\hline
\end{tabular}

\begin{tabular}{|c|c|c|c|c|}
\hline \multicolumn{4}{|c|}{ 存活率(\%) } & $\begin{array}{c}\mathrm{Mn} \\
/ \mathrm{umol} \cdot \mathrm{L}^{-1}\end{array}$ \\
\hline $100.0 \pm 3.5$ & $66.1 \pm 4.2^{*}$ & $72.2 \pm 3.4^{*}$ & $73.9 \pm 6.4^{*}$ & 0 \\
\hline $80.8 \pm 4.6^{*}$ & $86.7 \pm 7.0^{* \#}$ & $82.6 \pm 4.9^{*}$ & $73.3 \pm 3.1^{*}$ & 0.1 \\
\hline $76.9 \pm 3.9^{*}$ & $76.7 \pm 7.4^{*}$ & $69.3 \pm 4.4^{*}$ & $67.8 \pm 4.3^{* \#}$ & 1 \\
\hline $79.3 \pm 5.6^{*}$ & $58.1 \pm 10.8^{*}$ & $71.1 \pm 8.2^{*}$ & $80.2 \pm 8.6^{*}$ & 10 \\
\hline 0 & 0.06 & 0.6 & 6 & $\begin{array}{c}\text { 总黄酮 } \\
/ \mu \mathrm{g} \cdot \mathrm{mL}^{-1}\end{array}$ \\
\hline
\end{tabular}

图 3 药物合用对原代成骨细胞增殖的影响

(a) 淫羊蕉苷 $+\mathrm{Zn}$; (b) 总黄酮 $+\mathrm{Zn}$; (c) 淫羊雚苷 $+\mathrm{Ca}$; (d) 总黄酮 $+\mathrm{Ca}$; (e) 淫羊蕉苷 $+\mathrm{Mn}$; (f) 总黄酮 $+\mathrm{Mn}$. 成骨细胞增殖以 $A$ 样品 $/ A$ 空白 $\times 100 \%$ 表示, $A$ 空白设定为 $100 . *$ 表示与空白对照比较, $P<0.05 ;$ \#表示与淫羊蕉苷/总黄酮, 以及 $\mathrm{Zn} / \mathrm{Ca} / \mathrm{Mn}$ 比较, $P<0.05$

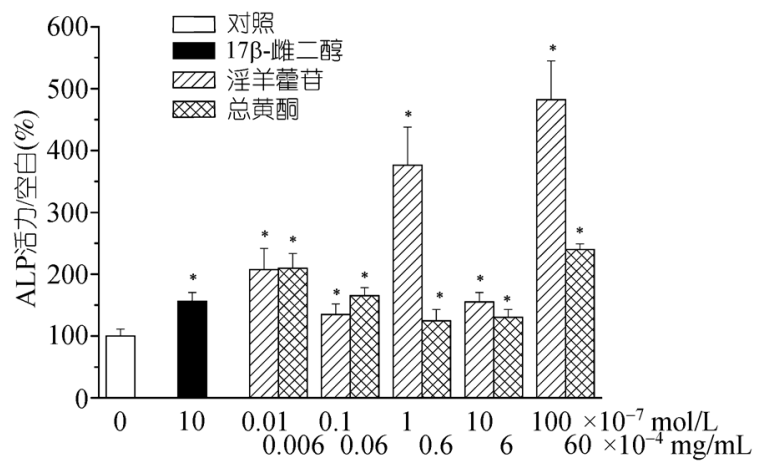

图 4 黄酮成分促进原代成骨细胞分化过程中 碱性磷酸酶的活力

$n=5, *$ 表示与空白对照组比, $P<0.05$

$7.1 ， 9.4 ， 5.7$ 和 3.6 倍, 而其单独作用时仅为 2.1 倍 $(\mathrm{Zn}), 3.8$ 倍(淫羊藿苷 $0.1 \mu \mathrm{mol} / \mathrm{L}$ ), 1.6 倍(淫羊藿苷 1 $\mu \mathrm{mol} / \mathrm{L}$ )， 4.8 倍(淫羊蕉苷 $10 \mu \mathrm{mol} / \mathrm{L}$ )， 1.2 倍(总黄酮 $0.06 \mu \mathrm{g} / \mathrm{mL}$ ), 1.3 倍(总黄酮 $0.6 \mu \mathrm{g} / \mathrm{mL}$ )和 2.4 倍(淫羊 蕉苷 $6 \mu \mathrm{g} / \mathrm{mL}$ ) (图 6(a)和(b)). 再如, 在前面的实验 中 $10 \mu \mathrm{mol} / \mathrm{L} \mathrm{Ca}$ 与 $0.1 \mu \mathrm{mol} / \mathrm{L} / /$ 淫羊蒦苷或总黄酮

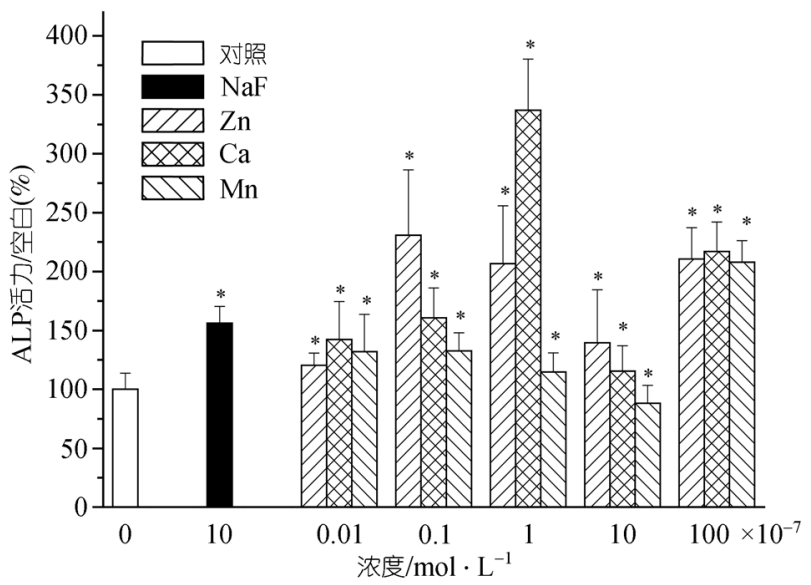

图 5 微量元素促进成骨细胞分化过程中碱性磷酸酶活力 $n=5$, * 表示与空白对照组比, $P<0.05$

$(0.6,6 \mu \mathrm{g} / \mathrm{mL})$ 合用显著减弱了黄酮成分对成骨细胞增 殖的抑制效应(图3(c) 和(d)), 显著促进了碱性磷酸酶活 力达 4.4, 3.5 和 2.6 倍(Ca 2.2 倍, 图 6(c)和(d)). 对于 $\mathrm{Mn}$ 而言, $10 \mu \mathrm{mol} / \mathrm{L} \mathrm{Mn}$ 分别与 10 和 $1 \mu \mathrm{mol} / \mathrm{L}$ 淫羊嚄苷合 用显示出明显的协同作用(图6(e)), 且前者的作用更加 强烈并与对成骨细胞增殖的影响相一致(图3(e)). 与此 
同时, 在某些淫羊蕉苷-Zn/Mn 合用以及总黄酮-Zn/ $\mathrm{Ca} / \mathrm{Mn}$ 合用时，成骨细胞的碱性磷酸酶活性有所降 低. 比如, $10 \mu \mathrm{mol} / \mathrm{L}$ 淫羊藿苷与 $1 \mu \mathrm{mol} / \mathrm{L} \mathrm{Zn}$ 合用, 以

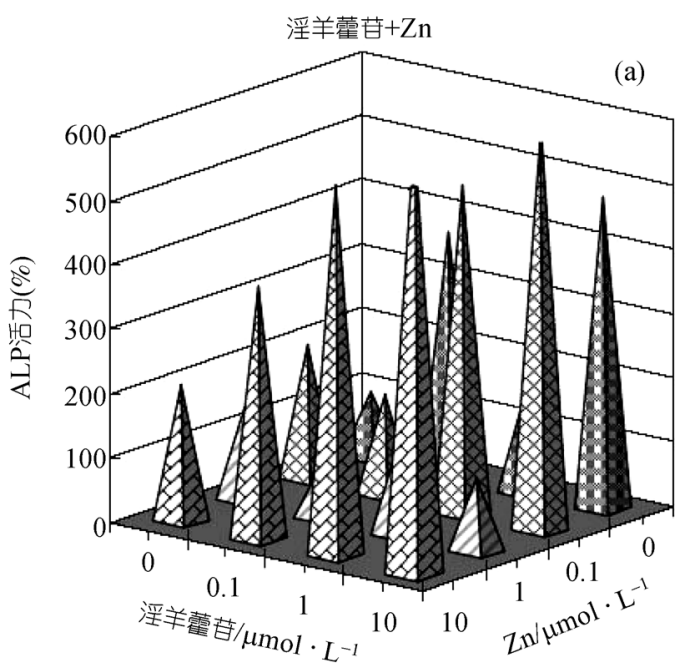

\begin{tabular}{|c|c|c|c|c|}
\hline \multicolumn{4}{|c|}{ ALP 活力(\%) } & $\begin{array}{c}\mathrm{Zn} \\
/ \mu \mathrm{mol} \cdot \mathrm{L}^{-1}\end{array}$ \\
\hline $100.0 \pm 8.6$ & $376.5 \pm 61.2^{*}$ & $155.1 \pm 15.2^{*}$ & $482.0 \pm 62.9^{*}$ & 0 \\
\hline $206.6 \pm 49.2^{*}$ & $156.0 \pm 11.9^{* \#}$ & $508.3 \pm 53.4^{* \#}$ & $628.2 \pm 34.8^{* \#}$ & 0.1 \\
\hline $139.6 \pm 44.8^{*}$ & $80.9 \pm 7.2^{* \#}$ & $143.5 \pm 7.8^{*}$ & $113.4 \pm 11.2^{* \#}$ & 1 \\
\hline $210.6 \pm 26.5^{*}$ & $391.8 \pm 28.9^{* \#}$ & $575.5 \pm 33.2^{* \#}$ & $705.8 \pm 40.4^{* \#}$ & 10 \\
\hline 0 & 0.1 & 1 & 10 & $\begin{array}{l}\text { 淫羊藿苷 } \\
/ \mu \mathrm{mol} \cdot \mathrm{L}^{-1}\end{array}$ \\
\hline
\end{tabular}

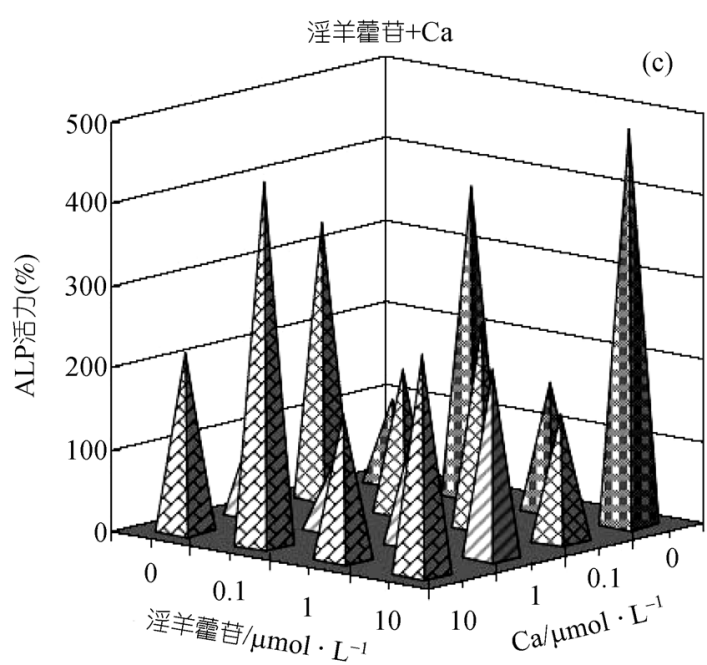

\begin{tabular}{|c|c|c|c|c|}
\hline \multicolumn{4}{|c|}{ ALP 活力 $(\%)$} & $\begin{array}{c}\mathrm{Ca} \\
\end{array}$ \\
\hline $100.0 \pm 9.3$ & $376.5 \pm 61.2^{*}$ & $155.1 \pm 15.2^{*}$ & $482.0 \pm 62.9^{*}$ & 0 \\
\hline $336.9 \pm 43.3^{*}$ & $175.2 \pm 6.3^{* \#}$ & $249.2 \pm 21.7^{*}$ & $155.3 \pm 9.7^{* \#}$ & 0.1 \\
\hline $110.6 \pm 21.2^{*}$ & $78.2 \pm 8.6^{* \#}$ & $127.8 \pm 7.9^{*}$ & $227.7 \pm 78.8^{*}$ & 1 \\
\hline $216.9 \pm 25.1^{*}$ & $444.0 \pm 32.4^{* \#}$ & $177.7 \pm 6.2^{*}$ & $267.2 \pm 13.4^{*}$ & 10 \\
\hline 0 & 0.1 & 1 & 10 & $\begin{array}{l}\text { 淫羊藿苷 } \\
/ \mu \mathrm{mol} \cdot \mathrm{L}^{-1}\end{array}$ \\
\hline
\end{tabular}

及 $0.06 \mu \mathrm{g} / \mathrm{mL}$ 总黄酮与 $10 \mu \mathrm{mol} / \mathrm{L} \mathrm{Mn}$ 合用, 在抑制成 骨细胞增殖的同时(图3(a)和(f)), 对成骨细胞的碱性磷 酸酶活力也显示出较强的抑制效应(图 6(a)和(f)).

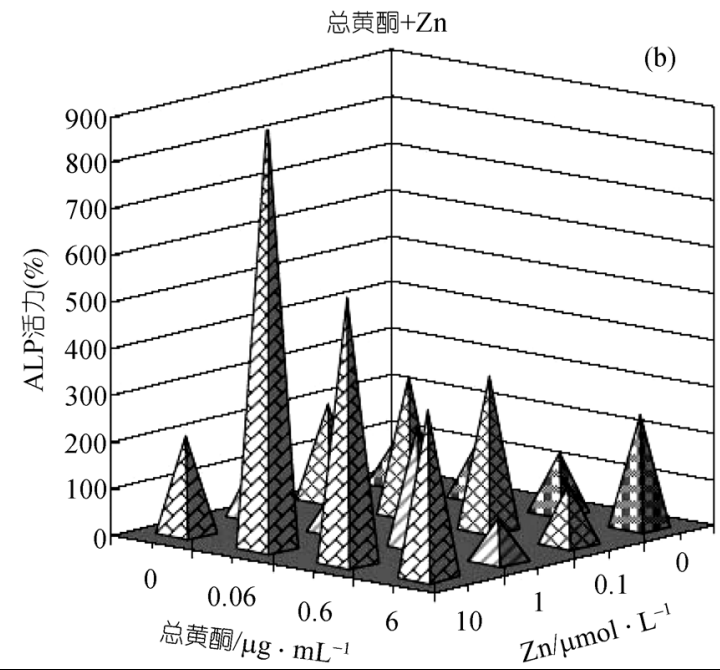

\begin{tabular}{|c|c|c|c|c|}
\hline \multicolumn{4}{|c|}{ ALP 活力 $(\%)$} & $\begin{array}{c}\mathrm{Zn} \\
/ \mu \mathrm{mol} \cdot \mathrm{L}^{-1}\end{array}$ \\
\hline $100.0 \pm 6.2$ & $124.6 \pm 18.4^{*}$ & $130.1 \pm 12.8^{*}$ & $239.9 \pm 9.3^{*}$ & 0 \\
\hline $206.6 \pm 49.2^{*}$ & $299.2 \pm 10.4^{* \#}$ & $327.5 \pm 15.2^{*}$ & $129.0 \pm 4.1^{* \#}$ & 0.1 \\
\hline $139.6 \pm 44.8^{*}$ & $94.0 \pm 5.3^{\#}$ & $256.1 \pm 10.5^{*}$ & $92.3 \pm 2.8^{\#}$ & 1 \\
\hline $210.6 \pm 26.5^{*}$ & $937.1 \pm 92.2^{* \#}$ & $568.2 \pm 13.4^{\#}$ & $360.4 \pm 24.5^{* \#}$ & 10 \\
\hline 0 & 0.06 & 0.6 & 6 & $\begin{array}{c}\text { 总黄酮 } \\
/ \mu \mathrm{g} \cdot \mathrm{mL}^{-1}\end{array}$ \\
\hline
\end{tabular}

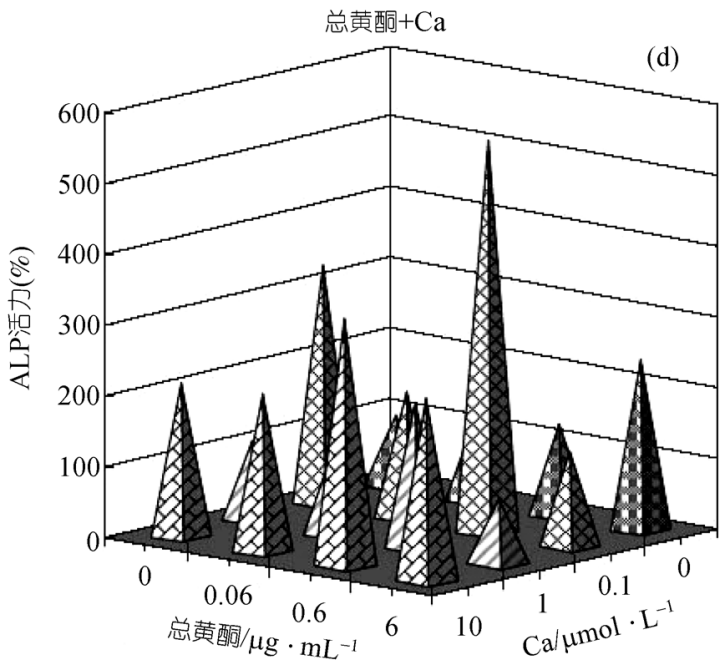

\begin{tabular}{|c|c|c|c|c|}
\hline \multicolumn{3}{|c|}{ ALP 活力 $(\%)$} & $\begin{array}{c}\mathrm{Ca} \\
/ \mu \mathrm{mol} \cdot \mathrm{L}^{-1}\end{array}$ \\
\hline $100.0 \pm 4.8$ & $124.6 \pm 18.4^{*}$ & $130.1 \pm 12.8^{*}$ & $239.9 \pm 9.3^{*}$ & 0 \\
\hline $336.9 \pm 43.3^{*}$ & $178.6 \pm 3.4^{*}$ & $553.4 \pm 24.6^{* \#}$ & $134.5 \pm 3.9^{* \#}$ & 0.1 \\
\hline $110.6 \pm 21.2^{*}$ & $121.2 \pm 3.2^{*}$ & $207.9 \pm 19.8^{* \#}$ & $91.2 \pm 2.6^{\#}$ & 1 \\
\hline $216.9 \pm 25.1^{*}$ & $222.6 \pm 3.4^{*}$ & $349.1 \pm 19.5^{* \#}$ & $259.4 \pm 15.5^{* \#}$ & 10 \\
\hline 0 & 0.06 & 0.6 & 6 & $\begin{array}{c}\text { 总黄酮 } \\
/ \mu \mathrm{g} \cdot \mathrm{mL}^{-1}\end{array}$ \\
\hline
\end{tabular}

(转下页) 

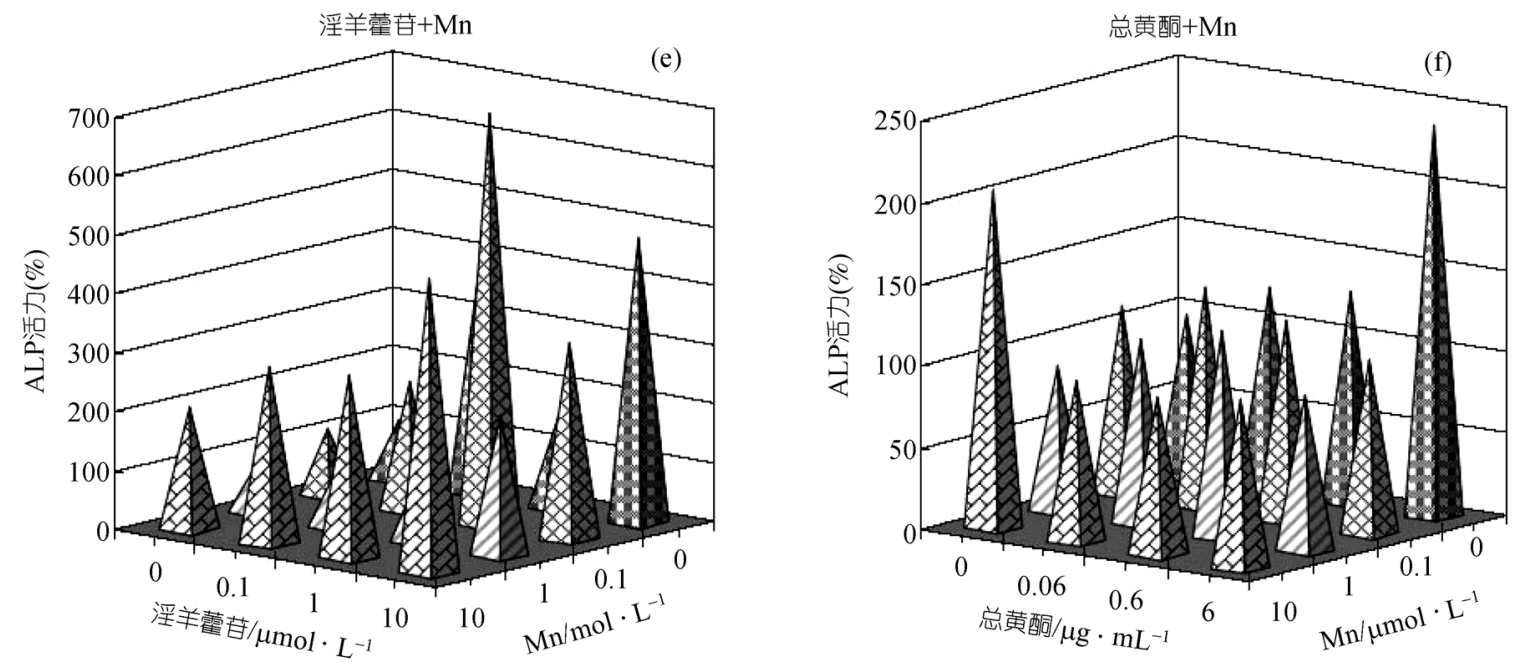

\begin{tabular}{|c|c|c|c|c|}
\hline \multicolumn{4}{|c|}{ ALP 活力 $(\%)$} & $\begin{array}{c}\mathrm{Mn} \\
\mu \mathrm{mol} \cdot \mathrm{L}^{-1}\end{array}$ \\
\hline $100.0 \pm 6.1$ & $376.5 \pm 61.2^{*}$ & $155.1 \pm 15.2^{*}$ & $482.0 \pm 62.9^{*}$ & 0 \\
\hline $114.8 \pm 16.1^{*}$ & $216.8 \pm 21.8^{*}$ & $696.2 \pm 50.3^{* \#}$ & $332.7 \pm 9.9^{*}$ & 0.1 \\
\hline $88.1 \pm 15.4^{*}$ & $104.1 \pm 10.2$ & $111.0 \pm 7.4^{*}$ & $237.0 \pm 5.9^{*}$ & 1 \\
\hline $207.8 \pm 18.4^{*}$ & $298.4 \pm 15.3^{*}$ & $308.5 \pm 14.9^{* \#}$ & $496.5 \pm 21.6^{* \#}$ & 10 \\
\hline 0 & 0.1 & 1 & 10 & $\begin{array}{c}\text { 淫羊嚾苷 } \\
/ \mu \mathrm{mol} \cdot \mathrm{L}^{-1}\end{array}$ \\
\hline
\end{tabular}

\begin{tabular}{|c|c|c|c|c|}
\hline \multicolumn{4}{|c|}{ ALP 活力 $(\%)$} & $\begin{array}{c}\mathrm{Mn} \\
/ \mu \mathrm{mol} \cdot \mathrm{L}^{-1}\end{array}$ \\
\hline $100.0 \pm 3.4$ & $124.6 \pm 18.4^{*}$ & $130.1 \pm 12.8^{*}$ & $239.9 \pm 9.3^{*}$ & 0 \\
\hline $114.8 \pm 16.1^{*}$ & $135.4 \pm 13.2^{*}$ & $122.2 \pm 11.5^{*}$ & $106.3 \pm 10.4$ & 0.1 \\
\hline $88.1 \pm 15.4^{*}$ & $113.5 \pm 8.2^{*}$ & $126.8 \pm 9.9^{*}$ & $94.9 \pm 7.4$ & 1 \\
\hline $207.8 \pm 18.4^{*}$ & $97.9 \pm 3.2^{\#}$ & $96.1 \pm 5.9^{\#}$ & $103.3 \pm 5.2^{\#}$ & 10 \\
\hline 0 & 0.06 & 0.6 & 6 & $\begin{array}{c}\text { 总黄酮 } \\
/ \mu \mathrm{g} \cdot \mathrm{mL}^{-1}\end{array}$ \\
\hline
\end{tabular}

图 6 淫羊蕉苷+微量元素以及总黄酮 + 微量元素对成骨细胞分化中碱性磷酸酶的活力显示出显著协同作用

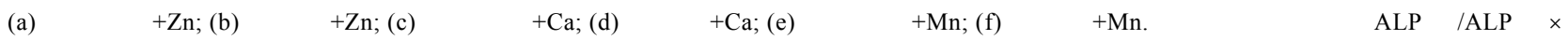
$100 \%$ 表示, $\mathrm{ALP}$ 空白 设定为 $100 . *$ 表示与空白对照比较, $P<0.05$; \#表示与淫羊雚苷/总黄酮, 以及 $\mathrm{Zn} / \mathrm{Ca} / \mathrm{Mn}$ 比较, $P<0.05$

\section{3 结论}

营养失调, 特别是微量元素和维生素缺乏, 在更 年期变得非常显著. 微量元素在骨基质合成过程中 发挥催化作用, 从而影响正常骨骼发育和更新 [24] 钙作为骨基质的主要组分, 在预防和治疗骨丢失中 备受关注; 同样, 其他的矿物元素在骨代谢和钙代谢 中也不容忽视. 有研究发现女性骨质疏松患者体内 血浆中钙、锌、锰的含量明显降低, 提示骨质疏松与 微量元素的缺乏存在一定联系 ${ }^{[19,25]}$. 此外, 临床研究 也证实, 补充 $\mathrm{Ca}, \mathrm{Zn}$ 和 $\mathrm{Mn}$ 可以有效提高绝经期后期 女性的脊椎骨骨矿物质密度 ${ }^{[26 ~ 28]}$.

根据中医药的观点, 人体发生疾病的根源在于 人体器官功能失调从而导致人体内部平衡的破坏 ${ }^{[29]}$. 实际上就其复杂性而言, 每味中药都是一个小复方, 其中多种成分通过复杂的机制恢复上述人体内部的 平衡. 多数中药在体内均显示出较好的疗效, 但是从 中药中提取分离得到的主要成分或粗提物针对体外 细胞模型的作用通常并不明显. 因此, 中药的临床效 果有可能存在多种不同的作用模式. 比如, i经过体内
代谢以活性代谢产物发挥作用, 或通过中药中各种 复杂成分之间的协同、加和等作用. 动物实验显示很 多中药对骨质疏松及其引起的骨折具有较好的治疗 作用 ${ }^{[2,3]}$. 可是骨碎补水提物(Drunaria fortunei)在体 外却显示出抑制成骨细胞增殖与分化的作用 ${ }^{[30]}$. 再 如, 淫羊蕉粗提物和黄酮成分在动物实验中显示出 较好的治疗和预防骨质疏松功效 ${ }^{[1 \sim 3,12]}$; 然而, 当其 作用于成骨细胞时并没有显示出应有的效果 ${ }^{[1]}$. 但是, 从给药阔叶淫羊蕉 (E. sagittatum) 的动物体内分离的 血清却显著促进成骨细胞的增殖和分化，从而提示 淫羊蕉中黄酮可能通过其活性代谢产物促进成骨细 胞的发育成熟 ${ }^{[1]}$.

本研究表明淫羊嚄苷/总黄酮与矿物元素 $(\mathrm{Zn}, \mathrm{Ca}$, $\mathrm{Mn})$ 合用对原代成骨细胞的存活率显示出较强的拮 抗作用, 而对成骨细胞分化中碱性磷酸酶活力则显 示出较强的协同效应. 如图 3 和 6 所示, $10 \mu \mathrm{mol} / \mathrm{L} \mathrm{Zn}$, $\mathrm{Ca}$ 或 $\mathrm{Mn}$ 与淫羊嚄苷/总黄酮合用减弱了黄酮成分对成 骨细胞增殖的抑制作用, 同时与黄酮成分和矿物元素 单独作用比较, 显著提高了成骨细胞的碱性磷酸酶活 力. 3 如 $10 \mu \mathrm{mol} / \mathrm{L} \mathrm{Zn}$ 与 $8.0 \times 10^{-7} \sim 1.0 \times 10^{-5} \mathrm{~mol} / \mathrm{L}$ 淫 
羊嚄苷, 或 $0.06 \sim 6 \mu \mathrm{g} / \mathrm{mL}$ 总黄酮合用在改善成骨细 胞存活率的同时, 显著增强了成骨细胞的碱性磷酸酶 活力, 分别达到 3.9, 5.8, 7.1, 9.4, 5.7 和 3.6 倍. 此外, $10 \mu \mathrm{mol} / \mathrm{L} \mathrm{Ca}$ 与 $0.1 \mu \mathrm{mol} / \mathrm{L}$ 淫羊蕉苷, 或 $0.6,6$ $\mu \mathrm{g} / \mathrm{mL}$ 总黄酮, $10 \mu \mathrm{mol} / \mathrm{L} \mathrm{Mn}$ 与 $10,1 \mu \mathrm{mol} / \mathrm{L}$ 淫羊蕉 甘合用显示出明显的协同作用, 并与对成骨细胞增殖 的影响相一致. 相反, $0.1 \mu \mathrm{mol} / \mathrm{L}, 1 \mu \mathrm{mol} / \mathrm{L} \mathrm{Zn}$ 与 10 $\mu \mathrm{mol} / \mathrm{L}$ 淫羊蕉苷, $10 \mu \mathrm{mol} / \mathrm{L} \mathrm{Mn}$ 与 $0.06 \mu \mathrm{g} / \mathrm{mL}$ 总黄酮 合用对成骨细胞的增殖则显示出更强的抑制效应. 同 时, 在某些淫羊蕉苷- $\mathrm{Zn} / \mathrm{Mn}$ 以及总黄酮 $-\mathrm{Zn} / \mathrm{Ca} / \mathrm{Mn}$ 合 用下也出现了对成骨细胞碱性磷酸酶活力促进率降低 的拮抗现象. 比如, 淫羊蕉苷 $(10 \mu \mathrm{mol} / \mathrm{L})-\mathrm{Zn}(1 \mu \mathrm{mol} / \mathrm{L})$ 以及总黄酮 $(0.06 \mu \mathrm{g} / \mathrm{mL})-\mathrm{Mn}(10 \mu \mathrm{mol} / \mathrm{L})$ 合用, 对成骨 细胞的增殖显示出更强的抑制效应，同时合用时与各 自对成骨细胞的分化的促进作用比较也有所降低. 综 上所述，实验结果显示黄酮成分与微量元素之间存在 着相互影响, 同时也显示中药中其他成分之间也存在 着潜在的相互影响, 而这些影响在一定程度上决定了 中药的最终功效. 总之, 中药预防和治疗骨质疏松的 作用机制非常复杂, 涉及到多种化学成分和各种微量 元素的作用, 有待于进一步的研究. 另外, 按照常规 “还原法”的研究思维, 通过化学分离和体外细胞模 型或生化指标, 期望找到可以代表中药药效的活性单 体, 通常结果是化学成分分离不少, 而真正的活性成 分却难以确定. 本文报道的结果表明, 可以按照中医 药的观点, 采用“组合法”, 即将几种化学成分或化 学成分与矿物元素的组合, 用来研究中药的作用物 质基础, 这也是本文希望提出的思考和探讨.

\section{参考文献}

1 Chen K M, Ge B F, Ma H P, et al. The serum of rats administered flavonoid extract from Epimedium sagittatum but not the extract itself enhances the development of rat calvarial osteoblast-like cells in vitro. Pharmazie, 2004, 59(1): 61-64

2 Hidaka S, Okamoto Y, Nakajima K. Preventive effects of traditional Chinese medicines on experimental osteoporosis induced by ovariectomy in rats. Calcif Tissue Int, 1997, 61: 239-246[DOI]

3 Huang H F, You J S. The use of Chinese herbal medicine on experimental fracture healing. Am J Chin Med, 1997, 25: 351$356[$ DOI]

4 Yeung, H C. Handbook of Chinese Herbs and Formulas. Los Angeles: Institute of Chinese Medicine, 1985

5 黄健, 张金超, 张天蓝, 等. 淫羊蕉苷对破骨细胞骨吸收功能的 影响及其作用机制. 科学通报, 2006, 51(24): 2851-2856

6 安胜军, 李桃, 李恩. 补肾方药对绝经后妇女卵巢功能和骨密度 的影响. 中国骨质疏松杂志, 2000, 6(2): 55-59

7 Wu H, Lien E J, Lien L L. Chemical and pharmacological investigations of Epimedium species: A survey. Prog Drug Res, 2003, 60: $51-57$

8 韩立民, 刘波, 徐彭. 淫羊蕉总黄酮对成骨细胞增殖的影响. 上
海中医药杂志, $2003,37(6): 55-57$

9 李勇, 季晖, 李萍, 等. 淫羊嚄总黄酮对体外培养成骨细胞的影 响. 中国药科大学学报, 2002, 33(1): 48-50

10 王俊勤, 胡有谷, 郑洪军, 等. 淫羊藿式对体外培养成骨细胞增 值和分化的影响. 中国临床康复, 2002, 6(9): 1307-1308

11 Meng F H, Li Y B, Xiong Z L, et al. Osteoblastic proliferative activity of Epimedium brevicornum Maxim. Phytomedicine, 2005, 12(3): 189-193[DOI]

12 Xie F, Wu C F, Lai W P, et al. The osteoprotective effect of Herba epimedii (HEP) extract in vivo and in vitro. eCAM, 2005, 2(3): 353361

13 Tucker K L. Dietary in take and bone status with aging. Curr Pharm Design, 2003, 9: 2687-2704[DOI]

14 Fujita T. Calcium bioavailability from heated oyster shell-seaweed calcium. Active absorbable algae calcium as assessed by urinary calcium excretion. J Bone Miner Metab, 1996, 14: 31-34[DOI]

15 Reid I R, Ames R W, Evans M C, et al. Effect of calcium supplementation on bone loss in postmenopausal women. N Engl J Med, 1993, 328: 460-464[DOI]

16 Beattie J, Avenell A. Trace element nutrition and bone metabolism. Nutr Res Rev, 1992, 5: 167-188[DOI]

17 Bray T M, Brettger W J. The physiological role of zinc as an antioxidant. Free Rad Biol Med, 1990, 8: 281—291[DOI]

18 Kishi S, Yamaguchi M. Inhibitory effect of zinc compounds on osteoblast-like cell formation in mouse marrow cultures. Biochem Pharmacol, 1994, 48: 1225-1230[DOI]

19 Relea P, Revilla M, Pipoll E, et al. Zinc, biochemical markers of nutrition, and type I osteoporosis. Age Aging, 1995, 24: 303307[DOI]

20 Keen C L, Zidenberg-Cherr S. Manganese. In: Ziegler E E, Filer L J, eds. Present Knowledge in Nutrition. 7th ed. Washington: ILSI Press, 1996

21 Zhang D W, Cheng Y, Zhang J C, et al. Effects of total flavonoids and flavonol glycosides from Epimedium koreanum Nakai on the proliferation and differentiation of primary osteoblasts. Phytomedicine, doi: 10.1016/j.phymed.2007.04.002

22 Chen T, Fry D. Hormonal regulation of the osteoblastic phenotype expression in neonatal murine calvarial cells. Calcif Tissue Int, 1999, 64: 304-309[DOI]

23 Carmichael J, Degraff W G, Gazdar A F, et al. Evaluation of a tetrazolium-based semiautomated colorimetric assay: assessment of chemosensitivity testing. Cancer Res, 1987, 47: 936-942

24 Gür A, Çolpan L, Nas K, et al. The role of trace minerals in the pathogenesis of postmenopausal osteoporosis and a new effect of calcitonin. J Bone Miner Metab, 2002, 20: 39-43[DOI]

25 Freeland-Graves J, Llanes C. Models to study manganese deficiency. In: Klimis-Tavantzis D L, ed. Manganese in Health and Disease. Boca Raton: CRC Press, Inc, 1994

26 Strause L, Saltman P, Smith K T, et al. Spinal bone loss in postmenopausal women supplemented with calcium and trace minerals. J Nutr, 1994, 124(7): 1060-1064

27 Rico H, Gomez-Raso N, Revilla M, et al. Effects on bone loss of manganese alone or with copper supplement in ovariectomized rats. A morphometric and densitomeric study. Eur J Obstet Gynecol Reprod Biol, 2000, 90(1): 97-101[DOI]

28 Nieves J W, Komar L, Cosman F, et al. Calcium potentiates the effect of estrogen and calcitonin on bone mass: Review and analysis. Am J Clin Nutr, 1998, 67: 18-24

29 Mo F Z. Multi-disciplinary research in traditional Chinese medicine - an exploration. Sci Found China, 2005, 13(2): 45-53

30 Liu H C, Chen R M, Jian W C, et al. Cytotoxic and antioxidant effects of the water extract of the traditional Chinese herb gusuibu (Drunaria fortunei) on rat osteoblasts. J Formos Med Assoc, 2001, 100(6): $383-388$ 